\title{
Near-Field Imaging of Interior Cavities
}

\author{
Peijun $\mathrm{Li}^{*}$ and Yuliang Wang \\ Department of Mathematics, Purdue University, West Lafayette, IN 47907, USA.
}

Received 1 April 2014; Accepted (in revised version) 25 September 2014

\begin{abstract}
A novel method is developed for solving the inverse problem of reconstructing the shape of an interior cavity. The boundary of the cavity is assumed to be a small and smooth perturbation of a circle. The incident field is generated by a point source inside the cavity. The scattering data is taken on a circle centered at the source. The method requires only a single incident wave at one frequency. Using a transformed field expansion, the original boundary value problem is reduced to a successive sequence of two-point boundary value problems and is solved in a closed form. By dropping higher order terms in the power series expansion, the inverse problem is linearized and an explicit relation is established between the Fourier coefficients of the cavity surface function and the total field. A nonlinear correction algorithm is devised to improve the accuracy of the reconstruction. Numerical results are presented to show the effectiveness of the method and its ability to obtain subwavelength resolution.
\end{abstract}

AMS subject classifications: 65N21, 78A46

Key words: Inverse scattering, near-field imaging, Helmholtz equation.

\section{Introduction}

Inverse scattering problems are concerned with determining the property of the medium by sending an incident wave and measuring the scattered field. These problems arise in a diverse range of applications, including radar, medical imaging, geophysical exploration, and non-destructive testing [12]. In this paper, we consider the problem of reconstructing the shape of an interior cavity by sending and receiving waves from inside. The cavity is represented by a simply connected domain in $\mathbb{R}^{2}$, and its exterior is assumed to be impenetrable. A time harmonic incident field is generated by a point source placed inside the cavity. Given the incident field, the direct problem is to determine the total field from the known cavity. We are mainly interested in the inverse problem, which is to reconstruct the shape of the cavity from the noisy data of the total field.

*Corresponding author. Email addresses: lipeijun@math.purdue.edu (P. Li), wang2049@math.purdue.edu (Y. Wang) 
The inverse problem was studied by using either the linear sampling method or the nonlinear integral equation method [23-26]. These work addressed conventional far-field imaging and the resolution was limited by the Rayleigh criterion [13]. Recently novel methods have been developed for solving a class of inverse surface scattering problems in the near-field imaging [3-6,10,17]. The surfaces are assumed to be small and smooth perturbations of planar surfaces. The methods require only a single incident field at one frequency, and are shown to be simple, efficient, and stable to reconstruct surfaces with subwavelength resolution. We refer to $[7,15]$ for near-field imaging of locally perturbed surfaces and $[1,2]$ for the resolution and stability analysis of a related wave imaging problem.

This work is an extension of near-field imaging from open surfaces to interior cavities. The boundary of the cavity is assumed to be a small and smooth perturbation of a circle centered at the origin. Using the Fourier series expansion, we derive a transparent boundary condition on an artificial circle inside the cavity, and formulate a boundary value problem for the total field. Based on the transformed field expansion, the problem is reduced to a successive sequence of two-point boundary value problems and is solved in a closed form. By dropping higher order terms in the power series expansion, the inverse problem is linearized and a simple reconstruction formula is obtained. Furthermore, a nonlinear correction algorithm is devised to improve the accuracy of the reconstruction. Numerical examples are presented to show the effectiveness of the method. The interior inverse cavity problem has been recently investigated in [27], where a decomposition method was studied to reconstruct the shape of the cavity by using the same data set, i.e., one point source and multiple measurements. The results in this paper are better than those reported in [27]. We refer to [8,16,18-22] for the application of transformed field expansion and related boundary perturbation methods for solving some direct scattering problems.

The paper is organized as follows. In Section 2, we introduce the model and formulation of the inverse problem. Section 3 is dedicated to the direct problem where the transformed field expansion is introduced to obtain an analytical solution. The reconstruction formula and the nonlinear correction algorithm are described in Section 4. Numerical examples are presented in Section 5. We conclude the paper in Section 6 with remarks and directions for future research.

\section{Model problem}

As seen in Fig. 1, the cavity is assumed to be a smooth perturbation of a disk and is defined by the domain

$$
\Omega=\{(r, \theta): 0 \leq r<a+f(\theta), \theta \in[0,2 \pi)\},
$$




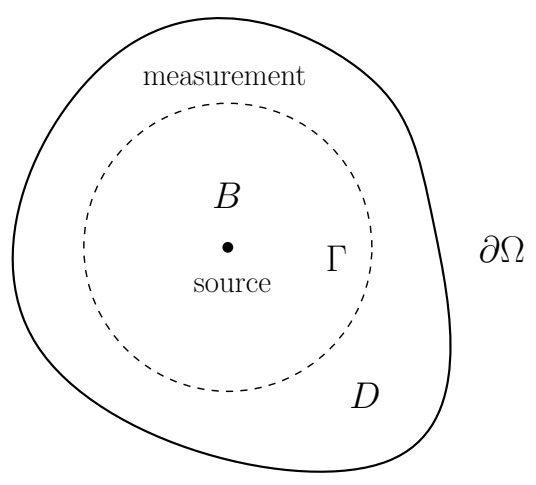

Figure 1: Problem geometry. The source is placed at the origin and the measurement is taken on the circle $\Gamma$.

where $a>0$ is the base radius and $f(\theta)$ is the cavity surface function. We assume that $f$ is smooth and small comparing to the wavelength $\lambda$ and can be written as

$$
f(\theta)=\varepsilon g(\theta),
$$

where $\varepsilon \ll \lambda$ is called the surface perturbation parameter and $g$ is the cavity profile function. A single point source is placed at the origin and generates the incident field

$$
u^{i}(r, \theta)=H_{0}^{(1)}(\kappa r) \quad \text { in } \Omega,
$$

where $H_{0}^{(1)}$ is the zero-th order Hankel function of the first kind and $\kappa=2 \pi / \lambda$ is the wavenumber. The total field $u$ satisfies one of the following boundary conditions:

$$
\begin{array}{rrrl}
\text { (I) sound soft: } & u=0 & & \text { on } \partial \Omega, \\
\text { (II) sound hard: } & \partial_{v} u=0 & & \text { on } \partial \Omega, \\
\text { (III) impedance: } & \left(\partial_{v}+\mathrm{i} \eta\right) u=0 & & \text { on } \partial \Omega,
\end{array}
$$

where $v$ is the unit outward normal vector on $\partial \Omega$ and $\eta>0$ is a given impedance constant.

Remark 2.1. Note that the impedance boundary condition (2.2c) reduces to the sound soft boundary condition (2.2a) when $\eta=\infty$, and reduces to the sound hard boundary condition (2.2b) when $\eta=0$. Hence we present the details for the impedance boundary condition and state the results for the other two boundary conditions without proofs.

Given the incident field and $f(\theta)$, the direct problem is to find the total field $u(r, \theta)$ in $\Omega$. It is well known from [9] that the direct problem has a unique solution in $H^{1}(\Omega)$ : if $\kappa^{2}$ is not a Dirichlet eigenvalue of $-\Delta$ in $\Omega$ for the sound soft boundary condition; if $\kappa^{2}$ is not a Neumann eigenvalue of $-\Delta$ in $\Omega$ for the sound hard boundary condition; for all values of $\kappa$ for the impedance boundary condition. We are interested in the inverse problem: to reconstruct the cavity surface function $f(\theta)$ from the noise total field $u_{\delta}(b, \theta)=u(b, \theta)+$ $\mathcal{O}(\delta)$ at the circle

$$
\Gamma=\{(r, \theta): r=b, \theta \in[0,2 \pi)\} \subset \Omega .
$$


Next we derive a transparent boundary condition on $\Gamma$. The scattered field $u^{s}$ satisfies the Helmholtz equation

$$
\left(\Delta+\kappa^{2}\right) u^{s}(r, \theta)=0 \quad \text { in } B
$$

where $B$ is the ball enclosed by $\Gamma$. It follows from the separation of variables that the solution admits the expansion

$$
u^{s}(r, \theta)=\sum_{n \in \mathbb{Z}} \alpha_{n} J_{n}(\kappa r) e^{\mathrm{i} n \theta} \quad \text { in } B,
$$

where $J_{n}$ is the $n$-th order Bessel function of the first kind. Note that the Bessel functions of the second kind are excluded in the expansion to ensure the boundedness of the scattered field.

Taking $\partial_{r}$ on both sides of (2.3) and comparing $\partial_{r} u^{s}(b, \theta)$ with $u^{s}(b, \theta)$ yield

$$
\left(\partial_{r}-T\right) u^{s}=0 \quad \text { on } \Gamma,
$$

where the linear operator $T$ is defined as

$$
\operatorname{Te} e^{\mathrm{i} n \theta}=\frac{\kappa J_{n}^{\prime}(\kappa b)}{J_{n}(\kappa b)} e^{\mathrm{i} n \theta}
$$

Taking $\left(\partial_{r}-T\right)$ on $u^{i}$ and using the Wronskian formula (e.g. [14]), we have

$$
\left(\partial_{r}-T\right) u^{i}=\frac{2 \mathrm{i}}{b \pi J_{0}(\kappa b)} \quad \text { on } \Gamma .
$$

Adding (2.4) and (2.5), we obtain the boundary condition

$$
\left(\partial_{r}-T\right) u=\frac{2 \mathrm{i}}{b \pi J_{0}(\kappa b)} \quad \text { on } \Gamma \text {. }
$$

Let $D$ be the domain bounded by $\partial \Omega$ and $\Gamma$, i.e.,

$$
D=\{(r, \theta): b<r<a+f(\theta), \theta \in[0,2 \pi)\} .
$$

It can be verified that the total field $u$ satisfies

$$
\left(\Delta+\kappa^{2}\right) u=0 \quad \text { in } D .
$$

Based on the Rayleigh-Faber-Krahn inequality, we have the following uniqueness result for the inverse problem with sound soft boundary condition. 
Theorem 2.1. Let $\Omega_{1}, \Omega_{2}$ be two sound soft cavities defined by the same base radius a and two surface functions $f_{1}(\theta), f_{2}(\theta)$ such that

$$
\kappa<j_{0,1} \sqrt{\frac{\pi}{A}}
$$

where $j_{0,1} \approx 2.4048$ is the first zero of $J_{0}$ and $A$ is the area enclosed between $\Omega_{1}, \Omega_{2}$, given explicitly by

$$
A=\frac{1}{2} \int_{0}^{2 \pi}\left|\left(a+f_{1}(\theta)\right)^{2}-\left(a+f_{2}(\theta)\right)^{2}\right| \mathrm{d} \theta .
$$

Let $b<\min _{j=1,2}\left\{a-\left\|f_{j}\right\|_{\infty}\right\}$ and $\Gamma$ be the circle with radius $b$ and center 0. Let $u_{1}(b, \theta), u_{2}(b, \theta)$ be the total field at $\Gamma$ for $\Omega_{1}, \Omega_{2}$ respectively. If $u_{1}(b, \theta)=u_{2}(b, \theta)$, then $f_{1}(\theta)=f_{2}(\theta)$.

Proof. Suppose $f_{1} \neq f_{2}$. Let $\Omega=\Omega_{1} \cap \Omega_{2}$. Then either $\Omega_{1} \backslash \Omega \neq \varnothing$ or $\Omega_{2} \backslash \Omega \neq \varnothing$. Without loss of generality, assume the former case and let $\Omega^{\prime}$ be a simply connected component of $\Omega_{1} \backslash \Omega$. Let $u=u_{1}-u_{2}$. Then $\left.u\right|_{\Gamma}=0$ and $\left.\partial_{\nu} u\right|_{\Gamma}=\left.T u\right|_{\Gamma}=0$. An application of the Holmgren's Theorem yields $u=0$ in $\Omega \backslash B$, and by unique continuation we obtain $u=0$ on $\partial \Omega$. In particular, we have $u=0$ on $\partial \Omega^{\prime} \cap \partial \Omega_{2}$. Hence $u_{1}=u_{2}=0$ on $\partial \Omega^{\prime} \cap \partial \Omega_{2}$. Besides we have $u_{1}=0$ on $\partial \Omega^{\prime} \cap \partial \Omega_{1}$. Hence $u_{1}=0$ on $\partial \Omega^{\prime}$. If follows from the uniqueness for the direct scattering problem and the Rayleigh-Faber-Krahn inequality that $u_{1}=0$ in $\Omega^{\prime}$ if (2.8) is satisfied. By unique continuation again we have $u_{1}=0$ in $\Omega_{1} \backslash B^{\prime}$, where $B^{\prime}$ is the ball with center 0 and radius $b^{\prime}<b$. In particular, we have $u=\partial_{\nu} u=0$ on $\Gamma$. This is a contradiction since $u_{1}$ satisfies (2.6).

Remark 2.2. The condition (2.8) is satisfied for sufficiently small $a$, for which we have global uniqueness. For the sound hard boundary condition, we have the same result except that $j_{0,1}$ in $(2.8)$ is replaced by $p_{1,1} \approx 1.8412$, the first positive zero of $J_{1}^{\prime}$. The uniqueness result holds globally for the impedance boundary condition.

\section{Transformed field expansion}

In this section we apply the transformed field expansion to solve analytically the direct scattering problem (2.7), (2.6), and (2.2).

Let $h=a-b$. We consider the change of variables

$$
\tilde{r}=\frac{h r+b f(\theta)}{h+f(\theta)}, \quad \tilde{\theta}=\theta, \quad \tilde{u}(\tilde{r}, \tilde{\theta})=u(r, \theta),
$$

which transforms the problem from the domain $D$ to the annulus $b<r<a$. A simple 
calculation yields

$$
\begin{aligned}
& \partial_{r}=\frac{h}{h+f} \partial_{\tilde{r}}, \quad \partial_{\theta}=-\frac{f^{\prime}}{h+f}(\tilde{r}-b) \partial_{\tilde{r}}+\partial_{\tilde{\theta}}, \quad \partial_{r r}=\left(\frac{h}{h+f}\right)^{2} \partial_{\tilde{r} \tilde{r}} \\
& \partial_{\theta \theta}=\left(\frac{f^{\prime}}{h+f}\right)^{2}(\tilde{r}-b)^{2} \partial_{\tilde{r} \tilde{r}}+\frac{2\left(f^{\prime}\right)^{2}-(h+f) f^{\prime \prime}}{(h+f)^{2}}(\tilde{r}-b) \partial_{\tilde{r}}-\frac{2 f^{\prime}}{h+f}(\tilde{r}-b) \partial_{\tilde{r} \tilde{\theta}}+\partial_{\tilde{\theta} \tilde{\theta}} .
\end{aligned}
$$

To simplify calculations, we apply another set of change of variables

$$
\begin{aligned}
& \hat{\tilde{r}}=\kappa \tilde{r}, \quad \hat{\tilde{\theta}}=\tilde{\theta}, \quad \hat{\tilde{u}}(\hat{\tilde{r}}, \hat{\tilde{\theta}})=\tilde{u}(\tilde{r}, \tilde{\theta}), \\
& \hat{a}=\kappa a, \quad \hat{b}=\kappa b, \quad \hat{f}=\kappa f, \quad \hat{g}=\kappa g, \quad \hat{h}=\kappa h, \quad \hat{\eta}=\frac{\eta}{\kappa},
\end{aligned}
$$

which normalize the problem with respect to the wavenumber. After a tedious but straightforward calculation, Eq. (2.7), after dropping the tildes and hats on the variables, becomes

$$
\left(c_{1} \partial_{r r}+c_{2} \partial_{r}+c_{3} \partial_{r \theta}+c_{4} \partial_{\theta \theta}+c_{5}\right) u(r, \theta)=0, \quad b<r<a,
$$

where

$$
\begin{aligned}
& c_{1}=h^{2}+2 h p f+p^{2}\left[f^{2}+\left(f^{\prime}\right)^{2}\right], \\
& c_{2}=\frac{1}{r}\left\{h^{2}+h\left[(p+1) f-p f^{\prime \prime}\right]+p\left[f^{2}-2\left(f^{\prime}\right)^{2}+f f^{\prime \prime}\right]\right\}, \\
& c_{3}=-\frac{2 p\left(h f^{\prime}+f f^{\prime}\right)}{r}, \\
& c_{4}=\frac{h^{2}+2 h f+f^{2}}{r^{2}}, \\
& c_{5}=h^{2}+2 h(p+1) f+\left(p^{2}+4 p+1\right) f^{2}+\frac{2\left(p+p^{2}\right) f^{3}}{h}+\frac{p^{2} f^{4}}{h^{2}},
\end{aligned}
$$

and the variable

$$
p=1-b / r
$$

is introduced to simplify the expressions.

Next we consider the boundary conditions (2.2) and (2.6). On a curve $r=r(\theta)$ we have

$$
\partial_{\nu} u=\frac{1}{\sqrt{r^{2}+\left(r^{\prime}\right)^{2}}}\left(r u_{r}-\frac{r^{\prime}}{r} u_{\theta}\right) .
$$

Substituting into the impedance boundary conditions (2.2c) and using the change of variables (3.1), (3.2), we obtain

$$
\begin{aligned}
& \left\{\left[h(a+f)^{2}+(h+f)\left(f^{\prime}\right)^{2}\right] \partial_{r}-(h+f) f^{\prime} \partial_{\theta}\right. \\
& \left.\quad+\mathrm{i} \eta(a+f)(h+f) \sqrt{(a+f)^{2}+\left(f^{\prime}\right)^{2}}\right\} u=0 \quad \text { on } r=a .
\end{aligned}
$$


Remark 3.1. Under the same change of variables, the sound soft boundary condition (2.2a) becomes

$$
u=0 \quad \text { on } r=a \text {, }
$$

and the sound hard boundary condition $(2.2 \mathrm{~b})$ becomes

$$
\left\{\left[h(a+f)^{2}+(h+f)\left(f^{\prime}\right)^{2}\right] \partial_{r}-(h+f) f^{\prime} \partial_{\theta}\right\} u=0 \quad \text { on } r=a,
$$

both of which can also be obtained from (3.4) by letting $\eta=\infty$ or $\eta=0$.

Similarly the transparent boundary condition (2.6) reduces to

$$
\partial_{r} u=\left(1+\frac{f}{h}\right)(T u+\rho) \quad \text { on } r=b,
$$

where $T$ is a linear operator satisfying

$$
T e^{\mathrm{i} n \theta}=\frac{J_{n}^{\prime}(b)}{J_{n}(b)} e^{\mathrm{i} n \theta}
$$

and

$$
\rho=\frac{2 \mathrm{i}}{b \pi J_{0}(b)} .
$$

Due to the small perturbation assumption (2.1), we consider a formal expansion of $u$ :

$$
u(r, \theta)=\sum_{m=0}^{\infty} \varepsilon^{m} u_{m}(r, \theta) .
$$

Substituting (3.8) and (2.1) into (3.3), and comparing the coefficients of the power series, we obtain the recurrence relation

$$
(\Delta+1) u_{m}=-v_{m},
$$

where

$$
v_{m}=\sum_{j=1}^{4} A_{j} u_{m-j},
$$

and

$$
\begin{aligned}
& A_{1}=\frac{1}{h}\left\{2 p g \partial_{r r}+\left[(p+1) g-p g^{\prime \prime}\right] \frac{\partial_{r}}{r}-2 p g^{\prime} \frac{\partial_{r \theta}}{r}+2 g \frac{\partial_{\theta \theta}}{r^{2}}+2(p+1) g\right\}, \\
& A_{2}=\frac{1}{h^{2}}\left\{p^{2}\left[g^{2}+\left(g^{\prime}\right)^{2}\right] \partial_{r r}+p\left[g^{2}-2\left(g^{\prime}\right)^{2}+g g^{\prime \prime}\right] \frac{\partial_{r}}{r}\right. \\
& \left.\quad-2 p g g^{\prime} \frac{\partial_{r \theta}}{r}+g^{2} \frac{\partial_{\theta \theta}}{r^{2}}+\left(p^{2}+4 p+1\right) g^{2}\right\}, \\
& A_{3}=\frac{2\left(p+p^{2}\right) g^{3}}{h^{3}}, \\
& A_{4}=\frac{p^{2} g^{4}}{h^{4}} .
\end{aligned}
$$


Substituting (3.8) and (2.1) into the boundary condition (3.4) yields

$$
\left(\partial_{r}+\mathrm{i} \eta\right) u_{m}=\varphi_{m} \quad \text { on } r=a ，
$$

where

$$
\varphi_{m}=\left[-\frac{2 g}{a} \partial_{r}+\frac{g^{\prime}}{a^{2}} \partial_{\theta}-\mathrm{i} \eta\left(\frac{1}{h}+\frac{2}{a}\right) g\right] u_{m-1}-\mathrm{i} \eta R_{m}
$$

and $R_{m}$ is a term which only contains $u_{m-j}$ for $j \geq 2$ :

$$
\begin{aligned}
R_{m}=\sum_{j=2}^{m} & \left\{\sum_{k=0}^{\lfloor j / 2\rfloor-1}\left(\frac{g^{2}+\left(g^{\prime}\right)^{2}}{a^{2}}\right)^{k}\left(\frac{2 g}{a}\right)^{j-2 k}\right. \\
& {\left.\left[C_{k, j-2 k}^{1 / 2}+\frac{1}{2}\left(\frac{a}{h}+1\right) C_{k, j-2 k-1}^{1 / 2}+\frac{a}{4 h} C_{k, j-2 k-2}^{1 / 2}\right]\right\} u_{m-j} . }
\end{aligned}
$$

Here $\lfloor n\rfloor$ denotes the largest integer not greater than $n$ and the generalized trinomial coefficient

$$
C_{k, l}^{1 / 2}=\frac{\frac{1}{2}\left(\frac{1}{2}-1\right) \cdots\left(\frac{1}{2}-k-l+1\right)}{k ! l !} .
$$

Remark 3.2. Using the same power series expansion, the sound soft boundary condition (3.5) yields

$$
u_{m}=0 \quad \text { on } r=a,
$$

and the sound hard boundary condition (3.6) yields

$$
\partial_{r} u_{m}=\varphi_{m} \quad \text { on } r=a,
$$

where

$$
\varphi_{m}=\left(-\frac{2 g}{a} \partial_{r}+\frac{g^{\prime}}{a^{2}} \partial_{\theta}\right) u_{m-1} .
$$

Again, (3.13) and (3.14) can be obtained from (3.11), (3.12) by setting $\eta=\infty$ and $\eta=0$ respectively.

Similarly the transparent boundary condition (3.7) reduces to

$$
\left(\partial_{r}-T\right) u_{m}=\rho_{m} \quad \text { on } r=b,
$$

where

$$
\rho_{0}=\rho, \quad \rho_{1}=\frac{g}{h}\left(T u_{0}+\rho\right), \quad \rho_{m}=\frac{g}{h}\left(T u_{m-1}\right), \quad m \geq 2 .
$$


It is understood that $u_{m}=0$ for $m<0$ in all recurrence relations.

Since $u_{m}, v_{m}, \varphi_{m}$, and $\rho_{m}$ are $2 \pi$-periodic functions in $\theta$, the boundary value problem (3.9), (3.11), (3.15) can be reduced to

$$
\begin{aligned}
& L_{n} u_{m}^{(n)}=-v_{m}^{(n)} \quad \text { in }(a, b), \\
& \left(\frac{\mathrm{d}}{\mathrm{d} r}+\mathrm{i} \eta\right) u_{m}^{(n)}=\varphi_{m}^{(n)} \quad \text { on } r=a, \\
& \left(\frac{\mathrm{d}}{\mathrm{d} r}-\frac{J_{n}^{\prime}(b)}{J_{n}(b)}\right) u_{m}^{(n)}=\rho_{m}^{(n)} \quad \text { on } r=b,
\end{aligned}
$$

where the superscript $(n)$ denotes the $n$-th Fourier coefficient and $L_{n}$ is the Bessel operator of order $n$. By Theorem B.1, we obtain the solution in the closed form

$$
u_{m}^{(n)}(r)=\Phi_{n}(r, b) \rho_{m}^{(n)}-\Phi_{n}(r, a) \varphi_{m}^{(n)}+\int_{a}^{b} \Phi_{n}(r, s) v_{m}^{(n)}(s) \mathrm{d} s, \quad r \in[a, b],
$$

where

$$
\begin{aligned}
& \Phi_{n}(r, s)=\frac{1}{W(s)} \begin{cases}J_{n}(r) E_{n}(s), & s<r, \\
J_{n}(s) E_{n}(r), & s>r,\end{cases} \\
& W(s)=W\left(J_{n}(s), Y_{n}(s)\right)=\frac{2}{\pi s}, \\
& E_{n}(z)=Y_{n}(z)-\frac{Y_{n}^{\prime}(a)+\mathrm{i} \eta Y_{n}(a)}{J_{n}^{\prime}(a)+\mathrm{i} \eta J_{n}(a)} J_{n}(z) .
\end{aligned}
$$

In particular, we may use (3.18) to obtain the closed forms for $u_{0}$ and $u_{1}$. By (3.10), (3.12) and (3.16) we have

$$
v_{0}^{(n)}=0, \quad \varphi_{0}^{(n)}=0, \quad \rho_{0}^{(n)}=\delta_{0 n} \rho,
$$

where $\delta_{0 n}$ is the Kronecker delta. Substituting into (3.18) yields

$$
u_{0}=\frac{\rho}{W(b)} J_{0}(b) E_{0}(r)=\mathrm{i} E_{0}(r) .
$$

It follows from (3.9) and (3.10) that

$$
\begin{aligned}
v_{1}=A_{1} u_{0} & =\frac{1}{h}\left\{2 p g \partial_{r r}+\left[(p+1) g-p g^{\prime \prime}\right] \frac{\partial_{r}}{r}+2(p+1) g\right\} u_{0} \\
& =\frac{1}{h}\left\{\left[(1-p) g-p g^{\prime \prime}\right] \frac{\partial_{r}}{r}+2 g\right\} u_{0},
\end{aligned}
$$

where we have used the fact that $u_{0}$ is independent of $\theta$ and satisfies the Bessel equation of order 0 . Taking the Fourier transform of $v_{1}$ in $\theta$ yields

$$
v_{1}^{(n)}=\frac{\mathrm{i}}{h}\left\{\left[1+\left(n^{2}-1\right) p\right] \frac{\partial_{r}}{r}+2\right\} E_{0}(r) g^{(n)}
$$


By (3.12) we have

$$
\varphi_{1}^{(n)}=\left[\eta\left(\frac{1}{h}+\frac{2}{a}\right) E_{0}(a)-\frac{2 \mathrm{i}}{a} E_{0}^{\prime}(a)\right] g^{(n)}=\frac{\eta}{h} E_{0}(a) g^{(n)} .
$$

By (3.16) we have

$$
\rho_{1}^{(n)}=\frac{1}{h}\left(\frac{J_{0}^{\prime}(b)}{J_{0}(b)} u_{0}(b)+\rho\right) g^{(n)}=\frac{\mathrm{i}}{h J_{0}(b)}\left(J_{0}^{\prime}(b) E_{0}(b)+W(b)\right) g^{(n)}
$$

Substituting (3.23), (3.24) into (3.18) and evaluating at $r=b$, we obtain

$$
u_{1}^{(n)}(b)=C_{n} g^{(n)}
$$

where

$$
\begin{aligned}
C_{n}=\frac{\mathrm{i} \pi}{2 h} J_{n}(b)\{ & \frac{b J_{0}^{\prime}(b) E_{0}(b)+\frac{2}{\pi}}{J_{0}(b)} E_{n}(b)+\mathrm{i} \eta a E_{0}(a) E_{n}(a) \\
& \left.+\int_{a}^{b} E_{n}(s)\left\{\left[1+\left(n^{2}-1\right) p(s)\right] E_{0}^{\prime}(s)+2 s E_{0}(s)\right\} \mathrm{d} s\right\} .
\end{aligned}
$$

Remark 3.3. Using Theorem B.2 and B.3, we can show (3.25) with

$$
\begin{aligned}
C_{n}=\frac{\mathrm{i} \pi}{2 h} J_{n}(b)\{ & \frac{b J_{0}^{\prime}(b) E_{0}(b)+\frac{2}{\pi}}{J_{0}(b)} E_{n}(b) \\
& \left.+\int_{a}^{b} E_{n}(s)\left\{\left[1+\left(n^{2}-1\right) p(s)\right] E_{0}^{\prime}(s)+2 s E_{0}(s)\right\} \mathrm{d} s\right\},
\end{aligned}
$$

where

$$
E_{n}=Y_{n}(z)-\frac{Y_{n}(a)}{J_{n}(a)} J_{n}(z)
$$

if $u$ satisfies the sound-soft boundary condition, and

$$
E_{n}=Y_{n}(z)-\frac{Y_{n}^{\prime}(a)}{J_{n}^{\prime}(a)} J_{n}(z),
$$

if $u$ satisfies the sound-hard boundary condition. 


\section{Reconstruction formula}

Based on (3.25), we now present the reconstruction formula. Let

$$
u_{\delta}(b, \theta)=u(b, \theta)+\mathcal{O}(\delta)
$$

be a noisy measurement of the total field at the circle $r=b$, where $\delta$ denotes the noise level. By the power series expansion (3.8) we obtain

$$
u_{\delta}(b, \theta)=u_{0}(b, \theta)+\varepsilon u_{1}(b, \theta)+\mathcal{O}\left(\varepsilon^{2}\right)+\mathcal{O}(\delta) .
$$

By Fourier series expansion in $\theta$ we have

$$
u_{\delta}^{(n)}(b)=u_{0}^{(n)}(b)+\varepsilon u_{1}^{(n)}(b)+\mathcal{O}\left(\varepsilon^{2}\right)+\mathcal{O}(\delta) .
$$

Substituting (3.25) and noticing $f^{(n)}=\varepsilon g^{(n)}$, we obtain

$$
f^{(n)}=S_{n}\left(u_{\delta}^{(n)}(b)-u_{0}^{(n)}(b)+\mathcal{O}\left(\varepsilon^{2}\right)+\mathcal{O}(\delta)\right), \quad S_{n}=C_{n}^{-1} .
$$

Dropping $\mathcal{O}\left(\varepsilon^{2}\right)$ and $\mathcal{O}(\delta)$ yields

$$
f^{(n)} \approx S_{n}\left(u_{\delta}^{(n)}(b)-u_{0}^{(n)}(b)\right) .
$$

Note that $u_{0}^{(n)}=\mathrm{i} E_{0}(b)$ by (3.22). Hence the right hand side of (4.2) consists of the measurement data and a known constant.

For the purpose of stability, we adopt the truncated Fourier series expansion

$$
f(\theta) \approx \sum_{|n| \leq N} f^{(n)} e^{\mathrm{i} n \theta}
$$

where $N$ is the cut-off frequency.

It follows from (4.1) that the ill-posedness of the inverse problem is determined by the behavior of $\left|S_{n}\right|$. In Fig. 2 we plot $\left|S_{n}\right|$ against $n$ for a model problem with base radius $a=1 \lambda$, impedance $\eta=2 \kappa$, and measurement height $h=0.1 \lambda, 0.2 \lambda, 0.3 \lambda$. Observe that $\left|S_{n}\right|$ stays relatively small for smaller $n$ and begins to increase at a roughly exponential rate for larger $n$. Plots for different values of $a$ indicate that the transition occurs at $n \approx$ $2 \pi a / \lambda$, the critical frequency for achieving subwavelength resolution. Hence it is stable to reconstruct the rough features above the wavelength and exponentially unstable to reconstruct the fine features below the wavelength. Moreover the growth rate of $\left|S_{n}\right|$ is lower for smaller $h$, which means the reconstruction is more stable and higher resolution can be achieved if the measurement is closer to the boundary. 


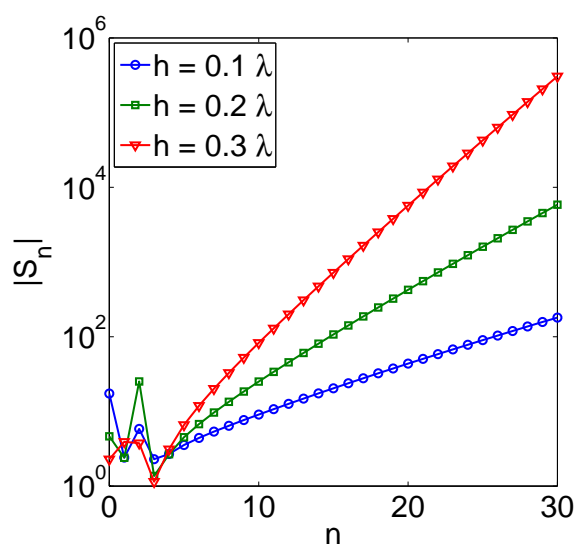

Figure 2: $\left|S_{n}\right|$ is plotted against $n$ for a model problem with $a=1 \lambda, \eta=2 \kappa$ and $h=0.1 \lambda, 0.2 \lambda, 0.3 \lambda$.

\subsection{Nonlinear correction}

It follows from (4.1) that the error in the reconstruction consists of two parts: the model error $\mathcal{O}\left(\varepsilon^{2}\right)$ originated from linearization and the data error $\mathcal{O}(\delta)$ introduced by measurement. In this section we present an iterative algorithm to correct the model error. We rewrite (4.1) as

$$
f^{(n)}=S_{n}\left(u_{\delta}^{(n)}(b)-u_{0}^{(n)}(b)+R_{n}(f)\right),
$$

where $R_{n}(f)=\mathcal{O}\left(\varepsilon^{2}\right)$ denotes the model error when the cavity surface function is $f$. Let $f_{0}$ be the initial guess reconstructed using (4.2) and (4.3). If $u_{f_{0}}$ is the total field with $f_{0}$ as the profile function, then we have

$$
f_{0}^{(n)}=S_{n}\left(u_{f_{0}}^{(n)}(b)-u_{0}^{(n)}(b)+R_{n}\left(f_{0}\right)\right) .
$$

Subtracting (4.4) from (4.1) and dropping the higher order error $R_{n}(f)-R_{n}\left(f_{0}\right)$, we have the updated Fourier coefficients

$$
f_{1}^{(n)}=f_{0}^{(n)}+S_{n}\left(u_{\delta}^{(n)}(b)-u_{f_{0}}^{(n)}(b)\right) .
$$

An updated cavity surface function $f_{1}$ is then obtained using (4.3) again. By repeating the above procedures, we have an iterative correction scheme:

$$
f_{k+1}^{(n)}=f_{k}^{(n)}+S_{n}\left(u_{\delta}^{(n)}(b)-u_{k}^{(n)}(b)\right),
$$

where $k$ denotes the iteration step and $u_{k}^{(n)}$ is the Fourier coefficient of the total field corresponding to the surface function $f_{k}$. 


\section{$5 \quad$ Numerical examples}

In this section we present numerical examples to show the effectiveness of our method. In all the examples we set the wavenumber $\kappa=2 \pi$ so that the wavelength $\lambda=1$. The base radius of the cavity is assumed to be $a=\lambda / 2$. As an example, we only report the results for the impedance boundary condition and the impedance is fixed at $\eta=2 \kappa$. The data is simulated by solving the direct problem with the integral equation method presented in Section A. The integral equation is discretized by dividing $[0,2 \pi)$ uniformly into 256 subintervals. The total field $u(b, \theta)$ is taken at 127 uniformly distributed angles in $[0,2 \pi)$. Relative noise is added to the data using

$$
u_{\delta}\left(b, \theta_{i}\right)=u\left(b, \theta_{i}\right)\left(1+\delta N_{i}\right),
$$

where $N_{i}$ are i.i.d. random variables following the standard normal distribution.

\subsection{Example one}

The profile function is given by $f(\theta)=\varepsilon g(\theta)$, where

$$
g(\theta)=0.5(\sin \theta+\sin 5 \theta) .
$$

Let $h=0.2 \lambda, \delta=0$ be fixed. Fig. 3 shows the reconstructed shape (dashed line) and the exact shape (solid line) of the cavity when the perturbation parameter $\varepsilon=0.05,0.1,0.15$. The dotted circle indicates where the measurement is taken in this and all the following figures. We have a nearly exact match when $\varepsilon=0.05$ and the result deteriorates as $\varepsilon$ increases. In particular, we achieved subwavelength resolution in all cases.

Next we fix $h=0.2 \lambda, \varepsilon=0.05$ and consider the effect of the noise level $\delta$. Fig. 4 shows the results for $\delta=0.01,0.05,0.1$ respectively. Clearly the accuracy decreases as the $\delta$ increases.

It can be seen from Fig. 2 that the inverse problem becomes more unstable as the measurement height $h$ increases. Fig. 5 shows the results of the reconstruction when
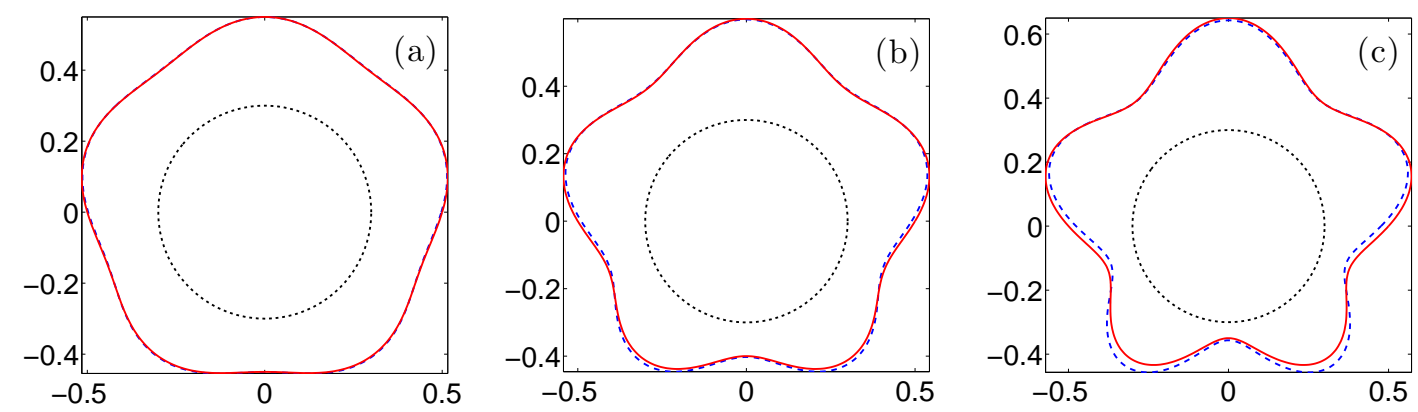

Figure 3: Example 1: Reconstructed shape (dashed line) and the exact shape (solid line) for $h=0.2 \lambda, \delta=0$ and (a) $\varepsilon=0.05$; (b) $\varepsilon=0.1$; (c) $\varepsilon=0.15$. 

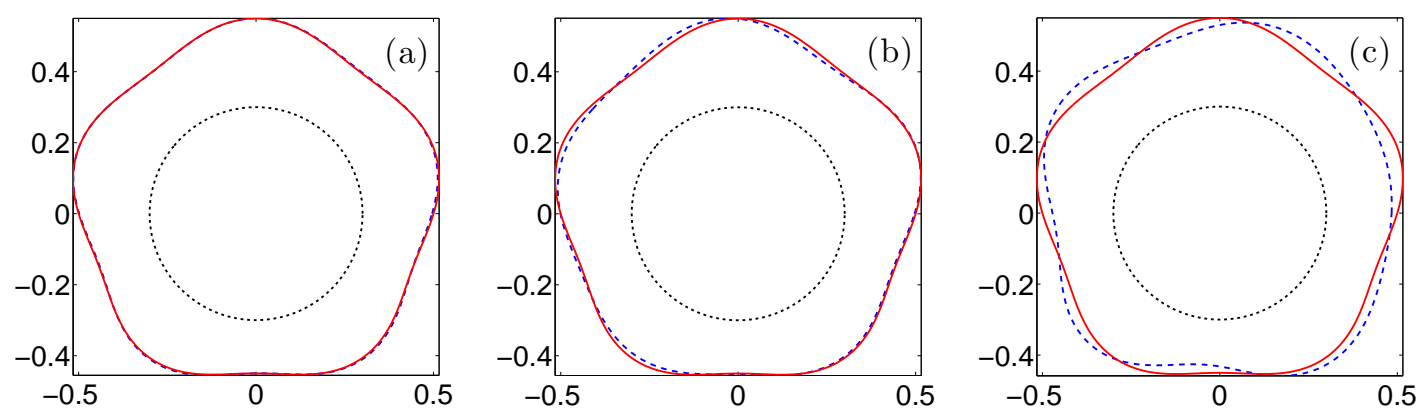

Figure 4: Example 1: Reconstructed shape (dashed line) and the exact shape (solid line) for $h=0.2 \lambda, \varepsilon=0.05$ and (a) $\delta=0.01$; (b) $\delta=0.05 ;$ (c) $\delta=0.1$.
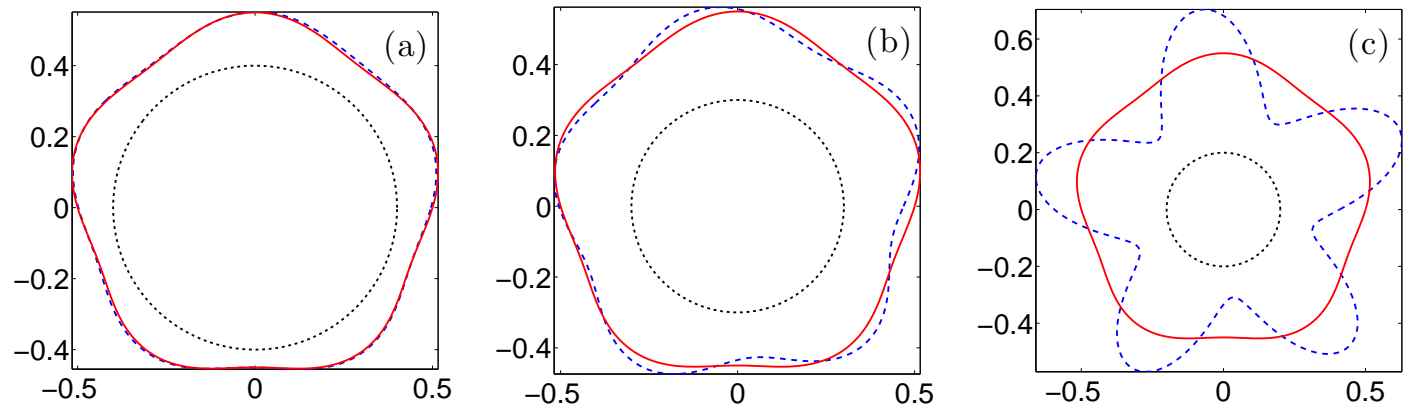

Figure 5: Example 1: Reconstructed shape (dashed line) and the exact shape (solid line) for $\varepsilon=0.05, \delta=0.05$ and (a) $h=0.1 \lambda$; (b) $h=0.2 \lambda$; (c) $h=0.3 \lambda$.
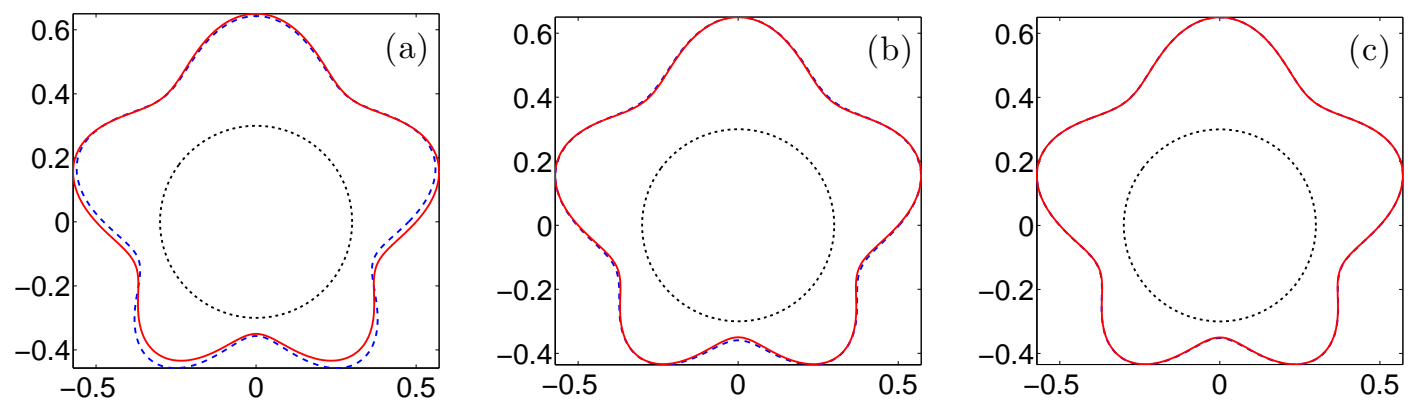

Figure 6: Example 1: Reconstructed shape (dashed line) and the exact shape (solid line) for $\varepsilon=0.15, h=0.2 \lambda, \delta=0$. (a) initial reconstruction; (b) after 3 iterations of nonlinear correction; (c) after 10 iterations of nonlinear correction.

$\varepsilon=0.05, \delta=0.05$ are fixed and $h$ is taken as $0.1 \lambda, 0.2 \lambda, 0.3 \lambda$ respectively. As anticipated the results deteriorates as $h$ increases.

Lastly we test the nonlinear correction algorithm presented in Section 4.1. Let $\varepsilon=$ $0.15, h=0.2 \lambda, \delta=0$ be fixed. Fig. 6 (a) shows the initial result of the reconstruction. No- 
tice some amplitude information is not completely resolved. After 3 iterations of the correction algorithm, we obtain the result in Fig. 6(b) and observe an evident improvement. Fig. 6(c) shows the result after 10 iterations and the shape is almost completely reconstructed. It is worthwhile to point out that our algorithm will converge only if $\varepsilon$ is sufficiently small. More advanced algorithms may be devised and is a direction for future research.

\subsection{Example two}

Let the exact profile function be given by $f(\theta)=\varepsilon g(\theta)$, where

$$
g(\theta)=e^{\sin 6 \theta} .
$$

This example is more difficult since the profile function contains infinitely many Fourier modes. Nevertheless the observations on the effect of the parameters $\varepsilon, h, \delta$ are similar. Thus we shall not present all the experiments but only the effect of $\varepsilon$ and the performance of the nonlinear correction. Let $h=0.2 \lambda, \delta=0$ be fixed. Fig. 7(a) and (b) shows the results for $\varepsilon=0.02$ and $\varepsilon=0.04$ respectively. Fig. 7(c) shows the result after 10 iterations of nonlinear correction with $\varepsilon=0.04$.
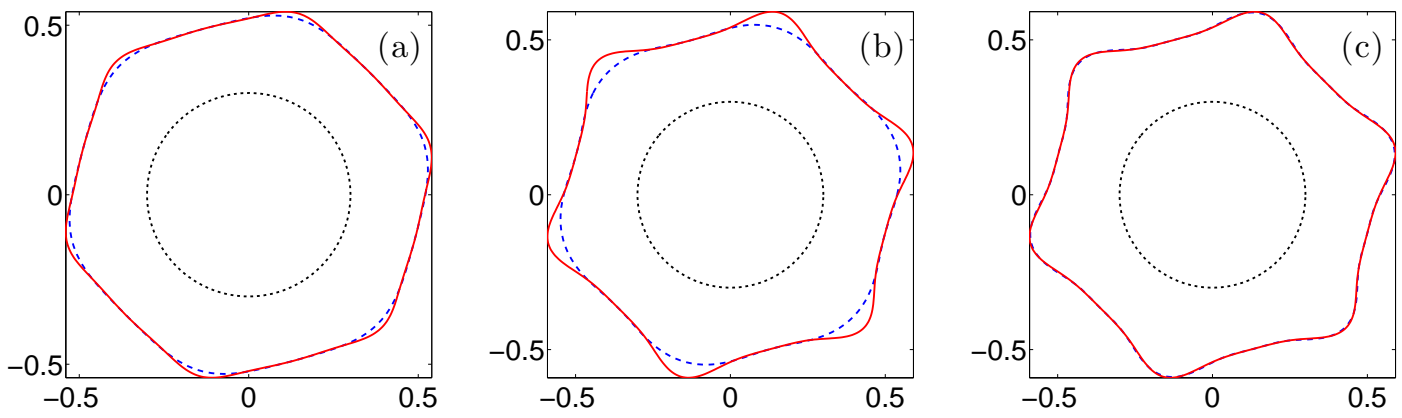

Figure 7: Example 2: Reconstructed shape (dashed line) and the exact shape (solid line) for $h=0.2 \lambda, \delta=0$. (a) $\varepsilon=0.02$; (b) $\varepsilon=0.04$, without correction; (c) $\varepsilon=0.04$, after 10 iterations of correction.

\section{Conclusion}

We considered the inverse problem of reconstructing the shape of an interior cavity by using a single point source and measurement data taken at a circle centered at the point source. The boundary of the cavity was assumed to be a small and smooth perturbation of a circle. We formulated a boundary value problem with sound soft, sound hard or impedance boundary condition on the boundary of the circle and a transparent boundary condition on the measurement circle. Using the transformed field expansion, we reduced the problem to a successive sequence of two-point boundary value problems 
and obtained the solutions in closed form. By dropping higher order terms in the power series expansion, we linearized the inverse problem and obtained the reconstruction formula: an explicit and simple relation between the Fourier modes of the data and the surface of the cavity. In addition, we proposed a nonlinear correction algorithm to improve the accuracy which is limited by the linearization. Numerical experiments showed that the method is simple, efficient, and stable to reconstruct the shape of cavities with subwavelength resolution.

As a future research project, we plan to extend the method to 3D cavities, where the full Maxwell equations should be considered. It is also worthwhile to relax the assumptions on the shape of the cavity. For example, we shall consider the case when the boundary can not be represented by a function but two parametric equations. We may also consider cavity that is not smooth, is a perturbation of more general shape, contains multiple scale or random features.

\section{Acknowledgments}

The research of PL was supported in part by the NSF grant DMS-1151308.

\section{A Integral equation method}

In this section we present an integral equation method for solving the direct cavity scattering problem. As before we normalize the problem with respect to the wavenumber $\kappa$ by the change of variables

$$
\hat{x}=\kappa x, \quad \hat{y}=\kappa y, \quad \hat{\eta}=\frac{\eta}{\kappa} .
$$

In the sequel we will work in the new variables, which are still denoted by $x, y, \eta$ for simplicity. Consider a cavity represented by a smooth domain $\Omega$ with $0 \in \Omega$. Let

$$
G(x, y)=\frac{\mathrm{i}}{4} H_{0}^{(1)}(|x-y|)
$$

be the Green's function for the Helmholtz equation. Let $u^{i}=H_{0}^{(1)}(|x|)$ be the incident field and $u^{\mathcal{S}}$ be the scattered field. Then we have

Lemma A.1.

$$
\begin{aligned}
& \int_{\partial \Omega} G(x, y) \partial_{\nu} u^{i}(y)-\partial_{v_{y}} G(x, y) u^{i}(y) \mathrm{d} s_{y}=0, \quad x \in \Omega, \quad x \neq 0, \\
& \int_{\partial \Omega} G(x, y) \partial_{\nu} u^{s}(y)-\partial_{v_{y}} G(x, y) u^{s}(y) \mathrm{d} s_{y}=u^{s}(x), \quad x \in \Omega .
\end{aligned}
$$


Proof. Let $x \in \Omega, x \neq 0$ be fixed. Denote by $B_{x, \sigma}, B_{0, \tau}$ two balls centered at $x, 0$ with radius $\sigma, \tau$ respectively. Let $\sigma, \tau$ be sufficiently small so that $B_{x, \sigma}, B_{0, \tau} \subset \Omega$ and $B_{x, \sigma} \cap B_{0, \tau}=\varnothing$. Since both $u^{i}(y)$ and $G(\cdot, y)$ satisfy the Helmholtz equation in $\Omega \backslash\left[B_{x, \sigma} \cup B_{0, \tau}\right]$, an application of the second Green's identity yields

$$
\int_{\partial \Omega \backslash\left[\partial B_{x, \sigma} \cup \partial B_{0, \tau}\right]} G(x, y) \partial_{\nu} u^{i}(y)-\partial_{v_{y}} G(x, y) u^{i}(y) \mathrm{d} s_{y}=0 .
$$

Straightforward calculations yield

$$
\partial_{v_{y}} G(x, y)=\frac{\mathrm{i}}{4} H_{1}^{(1)}(|x-y|), \quad y \in \partial B_{x, \sigma} .
$$

Using the asymptotic forms (e.g. [14])

$$
H_{0}^{(1)}(z) \sim \frac{2 \mathrm{i}}{\pi} \ln z, \quad H_{1}^{(1)}(z) \sim-\frac{2 \mathrm{i}}{\pi z} \quad \text { as } z \rightarrow 0,
$$

and the mean value theorem, we obtain

$$
\begin{aligned}
& \lim _{\sigma \rightarrow 0} \int_{\partial B_{x, \sigma}} G(x, y) \partial_{\nu} u^{i}(y)-\partial_{v_{y}} G(x, y) u^{i}(y) \mathrm{d} s_{y} \\
= & \lim _{\sigma \rightarrow 0} \frac{\mathrm{i}}{4} \int_{\partial B_{x, \sigma}} H_{0}^{(1)}(\sigma) \partial_{\nu} u^{i}(y)-H_{1}^{(1)}(\sigma) u^{i}(y) \mathrm{d} s_{y} \\
= & \frac{\mathrm{i}}{4}\left[\lim _{\sigma \rightarrow 0}\left(\frac{2 \mathrm{i}}{\pi} \ln (\sigma) 2 \pi \sigma\right) \partial_{\nu} u^{i}(x)+\lim _{\sigma \rightarrow 0}\left(\frac{2 \mathrm{i}}{\pi \sigma} 2 \pi \sigma\right) u^{i}(x)\right] \\
= & -u^{i}(x)=-H_{0}^{(1)}(|x|) .
\end{aligned}
$$

Similarly we have

$$
\lim _{\tau \rightarrow 0} \int_{\partial B_{0, \tau}} G(x, y) \partial_{\nu} u^{i}(y)-\partial_{\nu_{y}} G(x, y) u^{i}(y) \mathrm{d} s_{y}=H_{0}^{(1)}(|x|) .
$$

Taking $\lim _{\sigma \rightarrow 0, \tau \rightarrow 0}$ on (A.3) and using (A.4), (A.5) yields (A.1). The proof of (A.2) is similar and omitted.

Now we present the integral equation method for solving the boundary value problem (2.7), (2.6), (2.2).

Theorem A.1. Let $u=u^{i}+u^{s}$ be the total field.

1. If $u$ satisfies the sound soft boundary condition (2.2a), then

$$
u^{s}=\int_{\partial \Omega} G(x, y) \phi(y) \mathrm{d} s_{y}, \quad x \in \Omega,
$$

where $\phi(\cdot)=\left.\partial_{\nu} u\right|_{\partial \Omega}$ solves the integral equation

$$
\frac{\phi(x)}{2}-\int_{\partial \Omega} \partial_{\nu_{x}} G(x, y) \phi(y) \mathrm{d} s_{y}=\partial_{v} u^{i}(x), \quad x \in \partial \Omega .
$$


2. If $u$ satisfies the sound hard boundary condition (2.2b), then

$$
u^{s}=-\int_{\partial \Omega} \partial_{v_{y}} G(x, y) \phi(y) \mathrm{d} s_{y}, \quad x \in \Omega,
$$

where $\phi(\cdot)=\left.u\right|_{\partial \Omega}$ solves the integral equation

$$
\frac{\phi(x)}{2}+\int_{\partial \Omega} \partial_{v_{y}} G(x, y) \phi(y) \mathrm{d} s_{y}=u^{i}(x), \quad x \in \partial \Omega .
$$

3. If $u$ satisfies the impedance boundary condition (2.2c), then

$$
u^{s}=-\int_{\partial \Omega}\left[\partial_{v_{y}} G(x, y)+\mathrm{i} \eta G(x, y)\right] \phi(y) \mathrm{d} s_{y}, \quad x \in \Omega,
$$

where $\phi(\cdot)=\left.u\right|_{\partial \Omega}$ solves the integral equation

$$
\frac{\phi(x)}{2}+\int_{\partial \Omega}\left[\partial_{v_{y}} G(x, y)+\mathrm{i} \eta G(x, y)\right] \phi(y) \mathrm{d} s_{y}=u^{i}(x), \quad x \in \partial \Omega .
$$

Proof. Eq. (A.6) follows immediately by adding (A.1) to (A.2) and letting $\phi(y)=\partial_{v} u(y)$. Eq. (A.7) follows by taking $\partial_{v_{x}}$ on (A.6) and using the well known jump conditions for layer potentials. (see e.g. [11]). The other cases are similar.

\section{B A two-point boundary value problem}

In this section we present the solution method for the two-point boundary value problem (3.17).

Theorem B.1. Consider the following boundary value problem:

$$
\begin{aligned}
L_{n} u=-v \quad \text { in }(a, b), \\
\left(\frac{\mathrm{d}}{\mathrm{d} r}+\mathrm{i} \eta\right) u=\varphi \quad \text { on } r=a, \\
\left(\frac{\mathrm{d}}{\mathrm{d} r}-\frac{J_{n}^{\prime}(b)}{J_{n}(b)}\right) u=\rho \quad \text { on } r=b,
\end{aligned}
$$

where $L_{n}$ is the Bessel operator of order $n, b>a>0$ and $\eta \neq 0$. Then there exists a unique solution, which is given by

$$
u(r)=\Phi_{n}(r, b) \rho-\Phi_{n}(r, a) \varphi+\int_{a}^{b} \Phi_{n}(r, s) v(s) \mathrm{d} s, \quad r \in[a, b],
$$

where $\Phi_{n}$ is given by (3.19). 
Proof. Let $u_{1}, u_{2}$ be two solutions of (B.1), then $u=u_{1}-u_{2}$ satisfies the corresponding homogeneous boundary value problem

$$
\begin{array}{rlr}
L_{n} u=0 & \text { in }(a, b), \\
\left(\frac{\mathrm{d}}{\mathrm{d} r}+\mathrm{i} \eta\right) u=0 & \text { on } r=a, \\
\left(\frac{\mathrm{d}}{\mathrm{d} r}-\frac{J_{n}^{\prime}(b)}{J_{n}(b)}\right) u=0 & \text { on } r=b .
\end{array}
$$

A fundamental set of solutions for (B.3a) can be chosen as $\left\{J_{n}(r), Y_{n}(r)\right\}$, where $J_{n}$ and $Y_{n}$ are $n$-th order Bessel functions of the first and second kind, respectively. Hence we have $u=c_{1} J_{n}+c_{2} Y_{n}$ for some constants $c_{1}, c_{2}$. Using the boundary condition (B.3c) yields

$$
c_{2} \frac{W(b)}{J_{n}(b)}=0,
$$

where the Wronskian $W$ is given by (3.20). Since $W(b) \neq 0$, we have $c_{2}=0$. Using the boundary condition (B.3b) yields

$$
c_{1}\left[J_{n}^{\prime}(a)+\mathrm{i} \eta J_{n}(a)\right]=0 .
$$

Since $J_{n}(a)$ and $J_{n}^{\prime}(a)$ can not be zero simultaneously and $\eta \neq 0$, we have $c_{1}=0$. Hence $u=0$ and the uniqueness is established.

Now we return to the nonhomogeneous boundary value problem (B.1). Using variation of parameters, the general solution of the (B.1a) can be written as

$$
u(r)=c_{1} J_{n}(r)+c_{2} Y_{n}(r)+J_{n}(r) \int_{a}^{r} \frac{Y_{n}(s) v(s)}{W(s)} \mathrm{d} s-Y_{n}(r) \int_{a}^{r} \frac{J_{n}(s) v(s)}{W(s)} \mathrm{d} s,
$$

where the Wronskian $W$ is given explicitly by (3.20). Substituting (B.4) into the boundary condition (B.1b) yields

$$
c_{1}\left[J_{n}^{\prime}(a)+\mathrm{i} \eta J_{n}(a)\right]+c_{2}\left[Y_{n}^{\prime}(a)+\mathrm{i} \eta Y_{n}(a)\right]=\varphi .
$$

Substituting (B.4) into the boundary condition (B.1c) yields

$$
\frac{W(b)}{J_{n}(b)}\left(c_{2}-\int_{a}^{b} \frac{J_{n}(s) v(s)}{W(s)} \mathrm{d} s\right)=\rho .
$$

Solving (B.5), (B.6) for $c_{1}, c_{2}$, we obtain

$$
\begin{aligned}
& c_{1}=\frac{\varphi-c_{2}\left[Y_{n}^{\prime}(a)+\mathrm{i} \eta Y_{n}(a)\right]}{J_{n}^{\prime}(a)+\mathrm{i} \eta J_{n}(a)}, \\
& c_{2}=\frac{J_{n}(b) \rho}{W(b)}+\int_{a}^{b} \frac{J_{n}(s) v(s)}{W(s)} \mathrm{d} s .
\end{aligned}
$$


Substituting into (B.4) and rearranging, we have

$$
\begin{aligned}
u= & \frac{J_{n}(b) E_{n}(r)}{W(b)} \rho-\frac{J_{n}(r) E_{n}(a)}{W(a)} \varphi \\
& +E_{n}(r) \int_{a}^{b} \frac{J_{n}(s) v(s)}{W(s)} \mathrm{d} s+J_{n}(r) \int_{a}^{r} \frac{Y_{n}(s) v(s)}{W(s)} \mathrm{d} s-Y_{n}(r) \int_{a}^{r} \frac{J_{n}(s) v(s)}{W(s)} \mathrm{d} s
\end{aligned}
$$

where $E_{n}$ is given by (3.21). Now

$$
\begin{aligned}
& E_{n}(r) \int_{a}^{b} \frac{J_{n}(s) v(s)}{W(s)} \mathrm{d} s+J_{n}(r) \int_{a}^{r} \frac{Y_{n}(s) v(s)}{W(s)} \mathrm{d} s-Y_{n}(r) \int_{a}^{r} \frac{J_{n}(s) v(s)}{W(s)} \mathrm{d} s \\
= & E_{n}(r) \int_{r}^{b} \frac{J_{n}(s) v(s)}{W(s)} \mathrm{d} s+J_{n}(r) \int_{a}^{r} \frac{Y_{n}(s) v(s)}{W(s)} \mathrm{d} s+\left(E_{n}(r)-Y_{n}(r)\right) \int_{a}^{r} \frac{J_{n}(s) v(s)}{W(s)} \mathrm{d} s \\
= & E_{n}(r) \int_{r}^{b} \frac{J_{n}(s) v(s)}{W(s)} \mathrm{d} s+\int_{a}^{r} \frac{\left[\left(E_{n}(r)-Y_{n}(r)\right) J_{n}(s)+J_{n}(r) Y_{n}(s)\right] v(s)}{W(s)} \mathrm{d} s \\
= & E_{n}(r) \int_{r}^{b} \frac{J_{n}(s) v(s)}{W(s)} \mathrm{d} s+J_{n}(r) \int_{a}^{r} \frac{E_{n}(s) v(s)}{W(s)} \mathrm{d} s \\
= & \int_{a}^{b} \Phi_{n}(r, s) v(s) \mathrm{d} s,
\end{aligned}
$$

where $\Phi_{n}$ is given by (3.19). Substituting (B.8) into (B.7) and noticing

$$
\Phi_{n}(r, b)=\frac{J_{n}(b) E_{n}(r)}{W(b)}, \quad \Phi_{n}(r, a)=\frac{J_{n}(r) E_{n}(a)}{W(a)},
$$

we obtain (B.2).

Similarly we can solve the boundary value problem with sound soft or sound hard boundary conditions.

Theorem B.2. Consider the following boundary value problem:

$$
\begin{aligned}
L_{n} u=-v & \text { in }(a, b), \\
u=0 & \text { on } r=a, \\
\left(\frac{\mathrm{d}}{\mathrm{d} r}-\frac{J_{n}^{\prime}(b)}{J_{n}(b)}\right) u=\rho & \text { on } r=b,
\end{aligned}
$$

where $L_{n}$ is the Bessel operator of order $n$ and $b>a>0$. If $J_{n}(a) \neq 0$, then there exists a unique solution, which is given by

$$
u(r)=\Phi_{n}(r, b) \rho+\int_{a}^{b} \Phi_{n}(r, s) v(s) \mathrm{d} s, \quad r \in[a, b],
$$

where $\Phi_{n}$ is given by (3.19), in which $E_{n}$ is defined by (3.26). 
Theorem B.3. Consider the following boundary value problem:

$$
\begin{array}{rr}
L_{n} u=-v \quad & \text { in }(a, b), \\
\frac{\mathrm{d} u}{\mathrm{~d} r}=0 & \text { on } r=a, \\
\left(\frac{\mathrm{d}}{\mathrm{d} r}-\frac{J_{n}^{\prime}(b)}{J_{n}(b)}\right) u=\rho & \text { on } r=b,
\end{array}
$$

where $L_{n}$ is the Bessel operator of order $n$ and $b>a>0$. If $J_{n}^{\prime}(a) \neq 0$, then there exists a unique solution, which is given by

$$
u(r)=\Phi_{n}(r, b) \rho+\int_{a}^{b} \Phi_{n}(r, s) v(s) \mathrm{d} s, \quad r \in[a, b],
$$

where $\Phi_{n}$ is given by (3.19), in which $E_{n}$ is defined by (3.27).

\section{References}

[1] H. Ammari, J. Garnier, and K. Solna, Resolution and stability analysis in full-aperture, linearized conductivity and wave imaging, Proc. Amer. Math. Soc., 141 (2013), 3431-3446.

[2] H. Ammari, J. Garnier, and K. Solna, Limited view resolving power of linearized conductivity imaging from boundary measurements, SIAM J. Math. Anal., 45 (2013), 1704-1722.

[3] G. Bao, T. Cui and P. Li, Inverse diffraction grating of Maxwells equations in biperiodic structures, Optics Express, 22 (2014), 4799-4816.

[4] G. Bao and P. Li, Near-field imaging of infinite rough surfaces, SIAM J. Appl. Math., 73 (2013), 2162-2187.

[5] G. Bao and P. Li, Near-field imaging of infinite rough surfaces in dielectric media, SIAM J. Imaging Sci., 7 (2014), 867-899.

[6] G. Bao and P. Li, Convergence analysis in near-field imaging, Inverse Problems, to appear.

[7] G. Bao and J. Lin, Near-field imaging of the surface displacement on an infinite ground plane, Inverse Problems and Imaging, 7 (2013), 377-396.

[8] O. Bruno and F. Reitich, Numerical solution of diffraction problems: a method of variation of boundaries, J. Opt. Soc. Am. A, 10 (1993), 1168-1175.

[9] F. Cakoni and D. Colton, Qualitative Methods in Inverse Scattering Theory: An Introduction, Springer, 2006.

[10] T. Cheng, P. Li and Y. Wang, Near-field imaging of perfectly conducting grating surfaces, J. Opt. Soc. Am. A, 30 (2013), 2473-2481.

[11] D. Colton and R. Kress, Integral Equation Methods in Scattering Theory, Wiley, New York, 1983.

[12] D. Colton and R. Kress, Inverse Acoustic and Electromagnetic Scattering Theory, SpringerVerlag, Berlin, 1998.

[13] D. Courjon, Near-field Microscopy and Near-field Optics, Imperial College Press, London, 2003.

[14] NIST Digital Library of Mathematical Functions. http://dlmf .nist.gov/, Release 1.0.6 of 2013-05-06.

[15] G. Derveaux, G. Papanicolaou, and C. Tsogka, Resolution and denoising in near-field imaging, Inverse Problems, 22 (2006), 1437-1456. 
[16] P. Li and J. Shen, Analysis of the scattering by an unbounded rough surface, Math. Meth. Appl. Sci., 35 (2012), 2166-2184.

[17] P. Li and Y. Wang, Near-field imaging of obstacles, preprint.

[18] A. Malcolm and D. P. Nicholls, A field expansions method for scattering by periodic multilayered media, J. Acout. Soc. Am., 129 (2011), 1783-1793.

[19] A. Malcolm and D. P. Nicholls, A boundary perturbation method for recovering interface shapes in layered media, Inverse Problems, 27 (2011), 095009.

[20] D. P. Nicholls and F. Reitich, Shape deformations in rough surface scattering: cancellations, conditioning, and convergence, J. Opt. Soc. Am. A, 21 (2004), 590-605.

[21] D. P. Nicholls and F. Reitich, Shape deformations in rough surface scattering: improved algorithms, J. Opt. Soc. Am. A, 21 (2004), 606-621.

[22] D. P. Nicholls and J. Shen, A stable high-order method for two-dimensional boundedobstacle scattering, SIAM J. Sci. Comput., 28 (2006), 1398-1419

[23] H. Qin and F. Cakoni, Nonlinear integral equations for shape reconstruction in the inverse interior scattering problem, Inverse Problems, 27 (2011), 035005.

[24] H. Qin and D. Colton, The inverse scattering problem for cavities, Appl. Numer. Math., 62 (2012), 699-708.

[25] H. Qin and D. Colton, The inverse scattering problem for cavities with impedance boundary condition, Adv. Comput. Math., 36 (2012), 157-174.

[26] F. Zeng, F. Cakoni and J. Sun, An inverse electromagnetic scattering problem for a cavity, Inverse Problems, 27 (2011), 125002.

[27] F. Zeng, P. Suarez, and J. Sun, A decomposition method for an interior inverse scattering problem, Inverse Problems and Imaging, 7 (2013), 291-303. 